\title{
A Comparative Effectiveness Study of Two Oral Chemotherapy Drugs (UFT vs. Capecitabine) in Neoadjuvant Concurrent Chemoradiotherapy for Patients with Locally Advanced Rectal Cancer
}

\author{
CHUN-RU CHIEN ${ }^{1,2 *}$, WILLIAM TZU-LIANG CHEN ${ }^{2,3 *}$, HWEI-MIN WANG $^{3}$, TAO-WEI KE ${ }^{3}$, \\ HUA-CHE CHIANG ${ }^{3}$, SHENG-CHI CHANG ${ }^{3}$, YU-CHUN HUANG ${ }^{3}, \mathrm{CHE}^{-H U N G ~ L I N}{ }^{4}$, \\ CHUN-LIN HUANG ${ }^{5}$, YING-CHUN LIN ${ }^{1}$, TSUNG-WEI CHEN ${ }^{6}, \mathrm{CHIA-CHIN} \mathrm{LI}^{1}$ and K.S. CLIFFORD CHAO ${ }^{7}$ \\ Departments of ${ }^{1}$ Radiation Oncology, ${ }^{3}$ Colorectal Surgery and ${ }^{6}$ Pathology, \\ China Medical University Hospital, Taichung, Taiwan, R.O.C.; \\ ${ }^{2}$ School of Medicine, College of Medicine, China Medical University, Taichung, Taiwan, R.O.C.; \\ Divisions of ${ }^{4}$ Hematology and Oncology and ${ }^{5}$ Radiology, \\ China Medical University Hospital, Taichung, Taiwan, R.O.C.; \\ ${ }^{7}$ Cancer Center, China Medical University Hospital, Taichung, Taiwan, R.O.C.
}

\begin{abstract}
Background/Aim: Capecitabine is the current standard oral chemotherapy used in neoadjuvant concurrent chemoradiotherapy (NCCRT) for locally advanced rectal cancer (LARC) in North America. We compared the effectiveness of another oral chemotherapy agent, UFT (an oral combination of uracil and tegafur), to that of capecitabine. Materials and Methods: We identified LARC patients diagnosed from 2007 to 2011 using a populationbased registry in Taiwan (Health and Welfare Data Science Center, $H W D C$ ) and constructed a propensity score matched cohort to balance observable potential confounders. We compared the hazard ratio (HR) of death between the UFT and capecitabine groups. We performed supplementary analysis (SA) to evaluate the robustness of our finding regarding potential unobserved confounders (SA-1) and the robustness of the result in a subgroup when an additional potential confounder was taken into account (SA-2). Results:
\end{abstract}

This article is freely accessible online.

*These Authors contributed equally to this study.

Correspondence to: Chun-Ru Chien, MD, Ph.D., School of Medicine, College of Medicine, China Medical University, No.91 Hsueh-Shih Road, North District, Taichung 40402, Taiwan. Tel: +886 4220521217450, Fax: +886 4220521217460, e-mail: d16181@gmail.com

Key Words: Rectal cancer, neoadjuvant concurrent chemoradiotherapy, capecitabine, UFT.
Our study population comprised of 200 patients balanced with respect to observed co-variables. UFT lowered the hazard of death significantly more than capecitabine $(H R=0.58,95 \%$ confidence interval $(C I)=0.35-0.95$, $p=0.03)$. Our result was moderately sensitive in $S A-1$ but not significant in SA-2. Conclusion: The effectiveness of UFT in NCCRT for LARC is probably non-inferior to that of capecitabine.

Neoadjuvant concurrent chemoradiotherapy (NCCRT) is the current standard-of-care for locally advanced rectal cancer (LARC) $(1,2)$. The CAO/ARO/AIO-94 trial established the role of intravenous fluorouracil (FU) in NCCRT, although new drugs have also been extensively studied $(2,3)$.

Oral anti-cancer drugs have some potential merits that make them preferable to intravenous drugs. For example, oral anti-cancer drugs may make continuous drug exposure more feasible (4). Given this potential merit, an oral chemotherapy agent, capecitabine (a prodrug of 5fluorouracil (5-FU)), was found to have similar efficacy as intravenous fluorouracil and was recommended in the North American guidelines $(1,5)$. However, other oral chemotherapy agents are also available, such as UFT (an oral combination of uracil and tegafur in a fixed 1:4 molar ratio) (6), which was reported to have similar effects on survival as capecitabine for LARC patients receiving NCCRT in a European study (7).

We searched PubMed using the following keywords: "((rectal cancer neoadjuvant chemoradiotherapy) OR (rectal cancer preoperative chemoradiotherapy)) AND ((UFUR) OR 
(uracil-tegafur) OR (UFT) OR (uracil and tegafur))" on Mar 6th 2016 to find evidence regarding the efficacy of UFT for LARC patients receiving NCCRT, especially its efficacy relative to capecitabine. We did not find any head-to-head randomized studies comparing UFT and capecitabine in 91 search results, although an under-powered North American randomized study showed that UFT had similar survival rates but lower toxicity outcomes than intravenous FU (8). We also found the above-mentioned moderately sized $(n=112)$ European comparative effectiveness research (CER) (7) and other CERs that compared a UFT regimen to an intravenous chemotherapy drug regimen $(9,10)$. Given the limited evidence on this issue, we investigated the relative effectiveness of UFT and capecitabine in NCCRT for LARC patients via a propensity score (PS)-matched analysis among an Asian population.

\section{Materials and Methods}

Data source. The Health and Welfare Data Science Center (HWDC) database is a set of databases providing complete information from the Taiwan cancer registry (TCR) and death registry and reimbursement data for the whole Taiwan population provided by the Bureau of National Health Insurance (NHI) (11). The quality of this cancer registry was described in 2015 (12). The NHI is a singlepayer, compulsory social insurance program that provides insurance coverage to almost all citizens in Taiwan (13). All the above data were included in the HWDC with personal identifiers removed.

Case study identification and study design. The case study identification flow chart is shown in Figure 1. In brief, we identified clinical stage II - III rectal adenocarcinoma patients who received either UFT or capecitabine without intravenous chemotherapy during conventional fractionated NCCRT from 2007 to 2011. We used the date of diagnosis as the index date. We identified the explanatory variable of interest (UFT vs. capecitabine) using the reimbursement coding. We also collected other co-variables (including age, gender, period, comorbidity, clinical stage and radiotherapy dose) and outcome data from the HWDC. Finally, we constructed a PS-matched sample based on PSs estimated using the above co-variables to compare overall survival (OS). The final study population was exactly matched across two periods for the planned supplementary analysis (SA). In the PS analysis, we modeled the use of UFT ( $v s$. capecitabine) as the dependent variable and the above mentioned co-variables as independent variables and we used logistic regression to model the probability of receiving UFT. We then used the logit of the probability as the PS, as is typically used in the literature (14). We defined the co-variables based on our prior clinical- and HWDC-related research experiences (15-17). We defined the periods as 2007-2009 (6th American Joint Committee on Cancer (AJCC) staging) and 2010-2011 (7th AJCC staging). We further classified patients diagnosed between 2010 and 2011 into 2010 and 2011 groups (see "statistical and supplementary analysis" section). This study was approved by the Research Ethics Committee of our institution (CMUH103-REC-005).

Statistical and supplementary analysis. Tabulation and standardized differences were used to assess the balance of covariates between the
PS-matched groups. We compared the hazard ratio (HR) of death using Cox proportional hazards model with robust standard errors during the entire follow-up period (which ended on January 1st, 2015) for our primary analysis (18). Binary outcomes were compared via McNemar's test. In the planned SA-1, we examined the robustness of our finding given potential unmeasured confounder(s) as suggested in the literature because PS matching might not balance potential unmeasured confounder(s) (18). Under the assumption of "no unmeasured confounder," the probability of receiving either treatment should be the same after PS matching. However, if there was an unmeasured confounder associated with both treatment selection and outcome, then, the true probability of receiving treatment might differ for a factor (labelled as $\Gamma$ ), even after PS matching. This sensitivity analysis assessed the extreme statistical significance of the treatment effect that would be observed had this unmeasured confounder been accounted for, at various levels of $\Gamma$. Beginning in 2011, more information regarding prognosis (such as initial carcinoembryonic antigen (CEA) levels) and outcome (such as pathological response after NCCRT) was recorded in the TCR. Therefore, in the planned SA-2, we included the CEA level in the PS model in addition to the above-mentioned co-variables to construct a subgroup (those diagnosed in 2011) and then evaluated the OS (using the same method as in our primary analysis) and the degree of pathological response within this subgroup. SAS 9.3 (SAS Institute, Cary, NC, USA) was used for all analyses.

\section{Results}

Case study identification. As revealed in Figure 1, 586 clinical stage II-III rectal adenocarcinoma patients who received either UFT or capecitabine without intravenous chemotherapy during conventional fractionated NCCRT from 2007 to 2011 were identified as the initial study population. After excluding those with missing data and performing PS matching, the final study population included 200 patients. The characteristics of these patients are described in Table I. The balance of co-variables and small standardized differences $(<0.25)$ were good for all co-variables (19).

Primary analysis. We found that UFT was associated with a lower risk of death $(\mathrm{HR}=0.58,95 \%$ confidence interval $(\mathrm{CI})=0.35-0.95, p=0.03)$ than was capecitabine, as shown in Figure 2. The 5-year OS rates for the UFT and capecitabine groups were $75.13 \%$ and $53.75 \%$, respectively. The majority of patients received subsequent transabdominal resection (>95\% in both groups). The rate of abdominal-perineal resection (APR) and R0 resection for the UFT and capecitabine groups were also similar $(\leq 25 \%$ and $>95 \%$, respectively, both $p$-values $\geq 0.05$; however, the exact percentages are not reported per the HWDC guidelines due to the small sample size).

Supplementary analysis. In the SA-1, we found that the statistical significance of improved OS due to UFT was no longer significant if there was an unmeasured confounder that increased the odds of exposure by $15 \%$ (Table II). In the 
Step 1. Initial study population: From Cancer Registry ${ }^{1} 2007-2011$, we selected clinical stage II - III rectal adenocarcinoma patients who received either UFT or capecitabine without intravenous chemotherapy during conventional fractionated neoadjuvant concurrent chemoradiotherapy (NCCRT). We used the date of diagnosis as the index date $(\mathrm{n}=460(\mathrm{UFT})+126$ (capecitabine) $=586)$.

Step 2. Explanatory variable of interest (UFT or capecitabine) and other co-variables (age, gender, period, comorbidity, clinical stage ${ }^{2}$ and radiotherapy dose) were decided from cancer registry and reimbursement related files $(n=576$ after exclusion of missing data).

Step 3. Outcome variables: We used the death registry to evaluate overall survival (OS $)^{3}$.

Step 4. Final study population via propensity-score (PS) matching: We used the above co-variables to estimate the PS of receiving UFT ( $v s$. capecitabine) for each subject and used 1:1 PS matching among groups (UFT vs. capecitabine) to construct the final study population $(n=200)$.

Step 5. Analysis: We compared the hazard ratio of death with robust standard errors during the entire follow-up period. In supplementary analysis (SA-1), we examined the robustness of our finding under potential unmeasured confounder(s). In SA-2, we used the exacted matched subgroup (those diagnosed in 2011) to evaluate the impact after initial carcinoembryonic antigen level was taken into account.

${ }^{1}$ : We included only those treated by a single institution to ensure data consistency.

${ }^{2}:$ 2007-2009 patients were assessed using the $6^{\text {th }}$ American Joint Committee on Cancer staging, whereas 2010-2011 patients were assessed using the $7^{\text {th }}$ American Joint Committee on Cancer staging.

${ }^{3}$ : The exact number, after excluding the missing data, was not reported per the guidelines of the Health and Welfare Data Science Center due to the small sample size. 
Table I. Characteristics of the matched study population $(n=200)$.

\begin{tabular}{|c|c|c|c|}
\hline & $\begin{array}{c}\text { UFT } \\
\text { number }(\%) \\
\text { or mean }(\mathrm{sd})^{\mathrm{a}}\end{array}$ & $\begin{array}{c}\text { Capecitabine } \\
\text { number }(\%) \\
\text { or mean }(\mathrm{sd})^{\mathrm{a}}\end{array}$ & $\begin{array}{c}\text { Standardized } \\
\text { difference } \\
\text { (rounded) }\end{array}$ \\
\hline \multicolumn{4}{|l|}{ Age } \\
\hline$<65$ & $63(63)$ & $60(60)$ & 0.062 \\
\hline$>=65$ & 37 (37) & $40(40)$ & \\
\hline \multicolumn{4}{|l|}{ Gender } \\
\hline Female & $42(42)$ & $40(40)$ & 0.041 \\
\hline Male & $58(58)$ & $60(60)$ & \\
\hline \multicolumn{4}{|l|}{ Period } \\
\hline $2007-2009$ & $41(41)$ & $41(41)$ & 0 \\
\hline 2010 & $27(27)$ & $27(27)$ & \\
\hline 2011 & $32(32)$ & $32(32)$ & \\
\hline \multicolumn{4}{|l|}{ Comorbidity } \\
\hline Without $\mathrm{b}^{\mathrm{b}}$ & $73(73)$ & $68(68)$ & 0.11 \\
\hline With & $27(27)$ & $32(32)$ & \\
\hline \multicolumn{4}{|l|}{ Stage } \\
\hline II & $21(21)$ & $18(18)$ & 0.076 \\
\hline III & $79(79)$ & $82(82)$ & \\
\hline RT dose (Gy) & $48.87(2.30)$ & 48.39 (2.48) & 0.199 \\
\hline
\end{tabular}

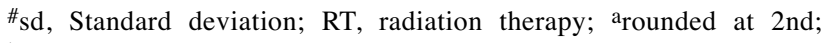
${ }^{\mathrm{b}}$ Carlson comorbidity score $=0$.

Table II. SA-1: Sensitivity analysis.

\begin{tabular}{ll}
\hline $\begin{array}{l}\text { Increased odds }(\Gamma) \text { of UFT } \\
\text { ( } v s \text {. capecitabine) by a hypothetic } \\
\text { unmeasured confounder }\end{array}$ & $p$-Value \\
\hline $10 \%$ & \\
$12 \%$ & 0.03606 \\
$14 \%$ & 0.04136 \\
$14.9 \%$ & 0.04717 \\
$15 \%$ & 0.04995 \\
\hline
\end{tabular}

SA-2, the balance of co-variables in this subgroup was good and had small standardized differences $(<0.25)$ (Table III). After the incorporation of the initial CEA level, the OS of the two groups became similar ( $p$-value $=0.9706$, Figure 3 ). The rates of pathological complete remission for UFT and capecitabine were also similar $(p$-value $=0.625)$.

\section{Discussion}

In this comparative effectiveness analysis, we found that OS was higher in LARC patients receiving NCCRT with UFT than in patients treated with capecitabine in the primary analysis of this population-based PS-matched analysis. However, this observation was only moderately sensitive in the sensitivity analysis. Furthermore, OS was similar in both
Table III. SA-2: Subgroup analysis for patients with known CEA level.

\begin{tabular}{lccc}
\hline & $\begin{array}{c}\text { UFT } \\
\text { number (\%) } \\
\text { or mean (sd) }\end{array}$ & $\begin{array}{c}\text { Capecitabine } \\
\text { number (\%) } \\
\text { or mean (sd) }\end{array}$ & $\begin{array}{c}\text { Standardized } \\
\text { difference } \\
\text { (rounded) }\end{array}$ \\
\hline Age & & & \\
$\quad<65$ & $21(65.63)$ & $22(68.75)$ & 0.067 \\
$>=65$ & $11(34.37)$ & $10(31.25)$ & \\
$\begin{array}{l}\text { Gender } \\
\text { Female } \\
\text { Male }\end{array}$ & $7(21.87)$ & $6(18.75)$ & 0.078 \\
$\begin{array}{l}\text { Comorbidity } \\
\text { Without }\end{array}$ & $25(78.13)$ & $26(81.25)$ & \\
$\quad \begin{array}{l}\text { With } \\
\text { Stage }\end{array}$ & $21(65.63)$ & $22(68.75)$ & 0.067 \\
$\quad$ II 5 & $11(34.37)$ & $10(31.25)$ & \\
$\quad$ III & $(15.62)$ & $5(15.62)$ & 0 \\
CEA level & & & \\
$\quad \begin{array}{l}\text { Above institution } \\
\text { normal level }\end{array}$ & $12(37.5)$ & $12(37.5)$ & 0 \\
$\quad \begin{array}{l}\text { Within institution } \\
\text { normal level }\end{array}$ & $20(62.5)$ & $20(62.5)$ & \\
RT dose (Gy) & $47.95(2.67)$ & $47.95(2.67)$ & 0 \\
\hline
\end{tabular}

\#sd, Standard deviation; RT, radiation therapy; CEA, carcinoembryonic

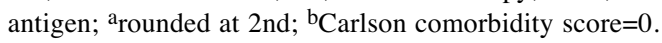

groups in the planned supplementary analysis when an additional co-variable was taken into account. Based on our findings, we feel that the effectiveness of UFT in NCCRT is probably non-inferior to capecitabine but further CERs and optimally randomized trials are needed to confirm this hypothesis.

We searched https://www.clinicaltrials.gov/ using the keyword "UFT" on Mar 20th, 2016. Among the 45 identified trials, we found no relevant trials, although there was a trial (NCT00207831) comparing NCCRT with UFT in neoadjuvant radiotherapy only for LARC patients that was terminated after the interim analysis. Therefore, there will probably be no level I evidence for the relative effectiveness of these drugs in the near future.

Although UFT outperformed capecitabine in our primary analysis, we felt that it might be better to conclude that "the effectiveness of UFT in NCCRT is probably non-inferior to capecitabine" based on the findings of our SA.

The result of our SA-2 was in line with the literature in that the OS was similar in both groups (7). In fact, another oral fluoropyrimidine anticancer agent (S1) was also reported to have similar effectiveness as capecitabine in another CER (20). In the era of personalized medicine, the use of molecular markers might be useful to predict potential responders when using these drugs (21).

Our results clearly demonstrated the potential limitations of CERs based on retrospective databases because we were 


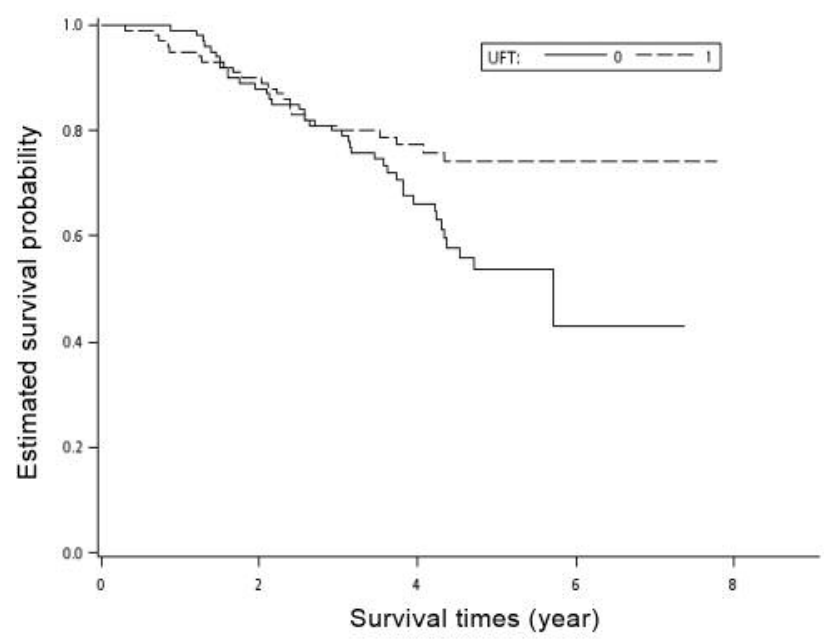

Figure 2. Kaplan-Meier survival curve (in years). UFT=0 (solid line) for capecitabine; UFT=1 (dotted line) for UFT.

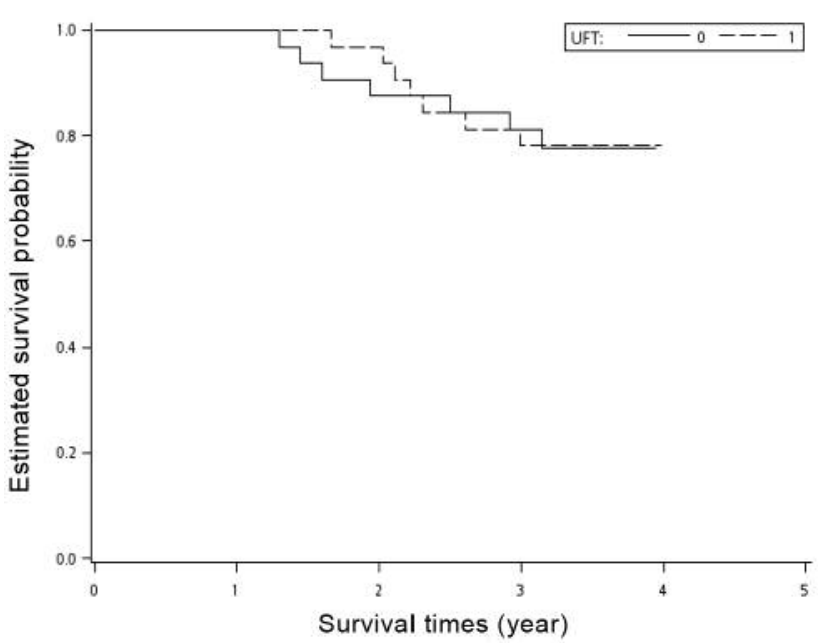

Figure 3. SA-2: Kaplan-Meier survival curve (in years). UFT=0 (solid line) for capecitabine; UFT=1 (dotted line) for UFT. unable to eliminate the impact of potential unobserved confounders. The result of our primary analysis was moderately sensitive in SA-1 and not supported in SA-2 when information for an additional potential confounder was available.

In conclusion, our study provided the first empirical evidence from Asia showing that the effectiveness of UFT in NCCRT for LARC is probably non-inferior to that of capecitabine. Our study results suggest that UFT might be a reasonable alternative in regions where UFT is available. Further CERs and optimally randomized trials are needed for confirmation.

\section{Acknowledgements}

The data analyzed in this study were provided by the Health and Welfare Data Science Center (HWDC) and the Ministry of Health and Welfare, Executive Yuan, Taiwan. We thank American Journal Experts (AJE) for their help in editing.

\section{References}

1 National Comprehensive Cancer Network (NCCN) Guidelines for Rectal Cancers, version 1.2016. Available at http:// www.nccn.org/professionals/physician_gls/pdf/rectal.pdf. Accessed March 16, 2016.

2 Sauer R, Liersch T, Merkel S, Fietkau R, Hohenberger W, Hess C, Becker H, Raab HR, Villanueva MT, Witzigmann H, Wittekind C, Beissbarth T and Rödel C: Preoperative versus postoperative chemoradiotherapy for locally advanced rectal cancer: results of the German CAO/ARO/AIO-94 randomized phase III trial after a median follow-up of 11 years. J Clin Oncol 30: 1926-1933, 2012.
3 Kye BH and Cho HM: Overview of radiation therapy for treating rectal cancer. Ann Coloproctol 30: 165-174, 2014.

4 Smieliauskas F, Chien CR, Shen C, Geynisman DM and Shih YC: Cost-effectiveness analyses of targeted oral anti-cancer drugs: a systematic review. Pharmacoeconomics 32: 651-680, 2014.

5 Hofheinz RD, Wenz F, Post S, Matzdorff A, Laechelt S, Hartmann JT, Müller L, Link H, Moehler M, Kettner E, Fritz E, Hieber U, Lindemann HW, Grunewald M, Kremers S, Constantin C, Hipp M, Hartung G, Gencer D, Kienle P, Burkholder I and Hochhaus A: Chemoradiotherapy with capecitabine versus fluorouracil for locally advanced rectal cancer: A randomized, multicentre, non-inferiority, phase 3 trial. Lancet Oncol 13: 579-588, 2012.

6 Hoff PM, Pazdur R, Benner SE and Canetta R: UFT and leucovorin: a review of its clinical development and therapeutic potential in the oral treatment of cancer. Anticancer Drugs 9: 479-490, 1998 .

7 Conde S, Borrego M, Teixeira T, Teixeira R, Sá A and Soares P: Comparison of neoadjuvant oral chemotherapy with UFT plus Folinic acid or Capecitabine concomitant with radiotherapy on locally advanced rectal cancer. Rep Pract Oncol Radiother 17: 376-383, 2012.

8 de la Torre A, García-Berrocal MI, Arias F, Mariño A, Valcárcel F, Magallón R, Regueiro CA, Romero J, Zapata I, de la Fuente C, Fernández-Lizarbe E, Vergara G, Belinchón B, Veiras M, Molerón R and Millán I: Preoperative chemoradiotherapy for rectal cancer: Randomized trial comparing oral uracil and tegafur and oral leucovorin $v s$. intravenous 5-fluorouracil and leucovorin. Int J Radiat Oncol Biol Phys 70: 102-110, 2008.

9 Díaz Beveridge R, Aparicio J, Tormo A, Estevan R, Artes J, Giménez A, Segura Á, Roldán S, Palasí R and Ramos D: Longterm results with oral fluoropyrimidines and oxaliplatin-based preoperative chemoradiotherapy in patients with resectable rectal cancer. A single-institution experience. Clin Transl Oncol 14: 471-480, 2012. 
10 Conde S, Borrego M, Teixeira T, Teixeira R, Sá A and Soares P: Neoadjuvant oral vs.. infusional chemoradiotherapy on locally advanced rectal cancer: Prognostic factors. Rep Pract Oncol Radiother 18: 67-75, 2012.

11 The Health and Welfare Data Science Center (HWDC) database. Available at http://www.mohw.gov.tw/cht/DOS/DM1.aspx? f_list_no=812. Accessed March 18, 2016 (in Chinese).

12 Chiang CJ, You SL, Chen CJ, Yang YW, Lo WC and Lai MS: Quality assessment and improvement of nationwide cancer registration system in Taiwan: A review. Jpn J Clin Oncol 45: 291-296, 2015.

13 Universal Health Coverage in Taiwan. Available at http://www.nhi.gov.tw/Resource/webdata/21717_1_20120808Un iversalHealthCoverage.pdf. Accessed March 20, 2016.

14 Austin PC, Chiu M, Ko DT, Goeree R and Tu JV: Propensity score matching for estimating treatment effect. In: Faries DE, Leon AC, Haro JM, Obenchain RL (eds.). Analysis of Observational Health Care Data Using SAS. USA: SAS Institute, Cary, NC, pp. 51-84, 2010.

15 Ke TW, Liao YM, Chiang HC, Chang SC, Wang PH, Chen YY, Chen WT and Chien CR: Effectiveness of neoadjuvant concurrent chemoradiotherapy versus up-front proctectomy in clinical stage II-III rectal cancer: A population-based study. Asia Pac J Clin Oncol 12: e234-40, 2016.

16 Fang HY, Hsiao FY, Huang HC, Lin YS, Chen CY, Shieh SH, Chen PR, Chen CK and Chien CR: Cost and effectiveness of video-assisted thoracoscopic surgery for clinical stage I nonsmall cell lung cancer: A population-based analysis. J Thorac Dis 6: 1690-1696, 2014.
17 Hsia TC, Tu CY, Fang HY, Liang JA, Li CC and Chien CR: Cost and effectiveness of image-guided radiotherapy for non-operated localized lung cancer: A population-based propensity scorematched analysis. J Thorac Dis 7: 1643-1649, 2015.

18 Austin PC: The use of propensity score methods with survival or time-to-event outcomes: Reporting measures of effect similar to those used in randomized experiments. Stat Med 33: 12421258, 2014.

19 Garrido MM, Kelley AS, Paris J, Roza K, Meier DE, Morrison RS and Aldridge MD: Methods for constructing and assessing propensity scores. Health Serv Res 49: 1701-1720, 2014.

$20 \mathrm{Su}$ M, Zhu LC, Wei HP, Luo WH, Lin RF and Zou CL: S-1Based versus capecitabine-based preoperative chemoradiotherapy in the treatment of locally advanced rectal cancer: a matched-pair analysis. PLoS One 9: e106162, 2014.

21 Sadahiro S, Suzuki T, Tanaka A, Okada K, Saito G, Kamijo A and Nagase H: Increase in Gene Expression of TYMP, DPYD and HIF1A Are Associated with Response to Preoperative Chemoradiotherapy Including S-1 or UFT for Rectal Cancer. Anticancer Res 36: 2433-40, 2016.
Received July 15, 2016

Revised August 18, 2016

Accepted August 19, 2016 